\title{
HYPERSURFACES WITH LIGHT-LIKE POINTS IN A LORENTZIAN MANIFOLD II
}

\author{
M. UMEHARA AND K. YAMADA
}

\begin{abstract}
In the authors' previous work, it was shown that if a zero mean curvature $C^{4}$-differentiable hypersurface in an arbitrarily given Lorentzian manifold admits a degenerate light-like point, then the hypersurface contains a light-like geodesic segment passing through the point. The purpose of this paper is to point out that the same conclusion holds with just $C^{3}$-differentiability of the hypersurfaces.
\end{abstract}

\section{INTRODUCTION}

This paper is a remark on authors' previous work [3]. We denote by $M$ a $C^{\infty}$-differentiable Lorentzian $(n+1)$-manifold $(n \geq 2)$. Let $U$ be a domain of $\left(\boldsymbol{R}^{n} ; u_{1}, \ldots, u_{n}\right)$ and $o(\in U)$ an arbitrary fixed point. We let $F: U \rightarrow M$ be a $C^{r}$-immersion $(r \geq 3)$. A point $q \in U$ which is neither time-like nor space-like is called a light-like point, which is a zero of the function $B_{F}$ defined in [3, (1.2)]. A light-like point $q$ is called degenerate if the exterior derivative of $B_{F}$ vanishes at $q$. In [3], a class $\mathcal{X}_{0}^{r}(M, \hat{o})$ of germs of $C^{r}$-immersion at $\hat{o}:=F(o)$ is defined. Zero mean curvature immersions in $M$ locally belong to this class. It was shown that:

Fact A ([3, Theorem A]). Suppose that $F \in \mathcal{X}_{0}^{r}(M, \hat{o})$ is a germ of a $C^{r}$-immersion $(r \geq 4)$ at a degenerate light-like point $o$. Then the image of $F$ contains a lightlike geodesic segment in $M$ passing through $\hat{o}(=F(o))$ consisting of only degenerate light-like points.

When $M=\boldsymbol{R}_{1}^{3}$ and $F$ is a zero mean curvature immersion, Fact $₫$ was shown by Klyachin 2 under the assumption that $F$ is $C^{3}$-differentiable. So it is expected that the fact also holds for any $C^{3}$-differentiable $F$. The above fact was applied to prove a Bernstein-type theorem in [1] for entire zero mean curvature graphs without time-like points. So by the following theorem, the main theorem of [1] holds under $C^{3}$-differentiability of the graphs:

Theorem B. In Fact $A$, the same conclusion holds even if $F$ is a germ of a $C^{3}$ immersion. In particular, if $F$ is a $C^{3}$-differentiable zero mean curvature hypersurface in $M$ containing a degenerate light-like point $o$, then $F$ contains a light-like geodesic segment passing through $F(o)$ consisting of only degenerate light-like points.

In [3], we proved Fact $\mathrm{A}$ by showing the local Lipschitz property of a certain system of ordinary differential equations (cf. [3, (4.8)]). However, by a careful tracing of the proof of 3 , Theorem A], we obtain the same conclusion under merely $C^{3}$-differentiability of $F$, as in the discussions in the next section. It should be

Date: March 27, 2020.

2010 Mathematics Subject Classification. Primary 53A10; Secondary 53B30, 35M10.

Key words and phrases. maximal hypersurface, light-like geodesic, zero mean curvature, Lorentzian manifolds.

The second author was partially supported by the Grant-in-Aid for Scientific Research (B) No. 17H02839 from Japan Society for the Promotion of Science. 
remarked that the authors do not know whether the theorem holds under $C^{2}$ differentiability of $F$ or not, which should remain an open problem.

\section{Proof of the ThEOREM}

Since $o \in U$ is a light-like point, there exists a non-zero tangent vector $\boldsymbol{v}$ of $U$ at $o$ such that $d F(\boldsymbol{v})$ is a light-like vector. We let $\sigma$ be a light-like geodesic passing through $F(o)$ such that $d F(\boldsymbol{v})$ points in the tangential direction of $\sigma$ at $F(o)$. We then take a Fermi-coordinate system $\left(x_{0}, \ldots, x_{n}\right)$ of signature $(-+\cdots+)$ centered at $F(o) \in M$ along $\sigma$ (cf. 33. Appendix A]). When $M$ is the LorentzMinkowski space $\boldsymbol{R}_{1}^{n+1}$, the Fermi coordinate system $\left(x_{0}, \ldots, x_{n}\right)$ can be taken as the canonical coordinate system.

Using this coordinate system, $\sigma$ can be expressed as

$$
\sigma(t)=(t, 0, \ldots, 0, t) .
$$

Moreover, all of the Christoffel symbols of the Lorentzian metric $g$ of $M$ vanish along $\sigma$. We can express $F$ as (cf. [3, (4.1)])

$$
\begin{aligned}
F\left(u_{1}, \ldots, u_{n}\right)=\left(f\left(x_{1}\left(u_{1}, \ldots, u_{n}\right), \ldots, x_{n}\left(u_{1}, \ldots, u_{n}\right)\right)\right. & \\
& \left.x_{1}\left(u_{1}, \ldots, u_{n}\right), \ldots, x_{n}\left(u_{1}, \ldots, u_{n}\right)\right),
\end{aligned}
$$

where $f\left(x_{1}, \ldots, x_{n}\right)$ is a $C^{3}$-function of variables $x_{1}, \ldots, x_{n}$ defined on a neighborhood of the origin $o \in \boldsymbol{R}^{n}$ ( $o$ corresponds to the degenerate light-like point of $F$ ). In this expression of $F,\left(x_{1}, \ldots, x_{n}\right)$ can be considered as a local coordinate system of $U$ at $o$ without loss of generality. We set $y:=x_{n}$ and use the notation ${ }^{\prime}=d / d y$, and set

(1.1) $a(y):=f(0, \ldots, 0, y), \quad$ and $\quad b_{i}(y):=f_{x_{i}}(0, \ldots, 0, y) \quad(i=1, \ldots, n-1)$,

where $f_{x_{i}}:=\partial f / \partial x_{i}$. Then $a$ (resp. $b_{i}$ for each $i=1, \ldots, n-1$ ) is a $C^{3}$ (resp. $C^{2}$ )-differentiable function of single variable $y$. Using these, we define a function $c$ by (cf. [3, (4.2)]) by

$$
f\left(x_{1}, \ldots, x_{n-1}, y\right)=a(y)+\left(\sum_{i=1}^{n-1} b_{i}(y) x_{i}\right)+c\left(x_{1}, \ldots, x_{n-1}, y\right) .
$$

Then $c$ is a function of class $C^{2}$, and the third derivatives

$$
c_{y x_{i} x_{j}}=c_{x_{i} y x_{j}}=f_{y x_{i} x_{j}}, \quad c_{y y x_{i}}=f_{y y x_{i}}-b_{i}^{\prime}
$$

exist for $i, j=1, \ldots, n-1$, which are continuous functions. By (1.1) and (1.2), one can easily show that

$$
\begin{aligned}
c(0, \ldots, 0, y) & =c_{x_{i}}(0, \ldots, 0, y)=c_{y}(0, \ldots, 0, y) \\
& =c_{y x_{i}}(0, \ldots, 0, y)=c_{y y x_{i}}(0, \ldots, 0, y)=0
\end{aligned}
$$

and that

$$
\begin{aligned}
f_{x_{i} x_{j} x_{k}}(0, \ldots, 0, y) & =c_{x_{i} x_{j} x_{k}}(0, \ldots, 0, y) \\
f_{y x_{i} x_{j}}(0, \ldots, 0, y) & =c_{y x_{i} x_{j}}(0, \ldots, 0, y)=c_{x_{i} y x_{j}}(0, \ldots, 0, y), \\
f_{y y x_{i}}(0, \ldots, 0, y) & =b_{i}^{\prime \prime}(y)
\end{aligned}
$$

for $i, j, k=1, \ldots, n-1$.

Remark 1. In [3] and [1], we used the function $h_{i j}$ satisfying

$$
f\left(x_{1}, \ldots, x_{n-1}, y\right)=a(y)+\sum_{i=1}^{n-1} b_{i}(y) x_{i}+\sum_{i, j=1}^{n-1} \frac{h_{i j}\left(x_{1}, \ldots, x_{n-1}, y\right)}{2} x_{i} x_{j} .
$$


Moreover, we wrote that $h_{i j}$ are $C^{1}$-functions. However it may not be true, in general. We should avoid this expression. In fact, for example, even if $h_{i i}$ is a $C^{1}$-function, the following computation is not allowed

$$
\left(h_{i i} x_{i}^{2}\right)_{x_{i} x_{i}}=\left(h_{i i}\right)_{x_{i} x_{i}} x_{i}^{2}+2\left(h_{i i}\right)_{x_{i}} x_{i}+2 h_{i i} .
$$

So it should be replaced (1.5) by (1.2) in [3] and the final remark of [1].

The condition that $o$ is a degenerate light-like point is written as (cf. [3, (4.3) and (4.5)])

$$
a(0)=0, \quad a^{\prime}(0)=1, \quad b_{i}(0)=b_{i}^{\prime}(0)=0 \quad(i=1, \ldots, n-1),
$$

where $^{\prime}=d / d y$.

Since $F \in \mathcal{X}_{0}^{3}(M, \hat{o})$, there exists a $C^{1}$-function $\varphi$ such that (cf. 3. (3.1) and (3.3)])

$$
\tilde{A}:=A-\varphi B
$$

vanishes identically on $U$, where

$$
B:=B_{F}, \quad A:=A_{F}
$$

are functions associated with $F$ defined in [3, (2.4) and (2.6)]. We remark that $B, \nabla B$ and $A$ can be expressed without use of derivatives of the Christoffel symbols, where $\nabla B:=\left(B_{x_{1}}, \ldots, B_{x_{n}}\right)$. Since $\left(x_{0}, \ldots, x_{n}\right)$ is a Fermi coordinate system, the expressions of $B, \nabla B$ and $A$ along $\sigma$ coincide with those of $\boldsymbol{R}_{1}^{n+1}$ given in [3, Appendix B]. For the Lorentzian metric $g$ of $M$, set

$$
g_{\alpha \beta}:=g\left(\partial_{x_{\alpha}}, \partial_{x_{\beta}}\right), \quad \hat{g}_{\alpha \beta}:=g_{\alpha \beta} \circ F \quad(\alpha, \beta=0, \ldots, n),
$$

where $\partial_{x_{\alpha}}:=\partial / \partial x_{\alpha}$. Since we can write

$$
F_{x_{i}}=f_{x_{i}} \partial_{0}+\partial_{x_{i}} \quad(i=1, \ldots, n),
$$

the coefficients $s_{i, j}$ of the induced metric $d s^{2}$ with respect to the local coordinate system $\left(U ; x_{1}, \ldots, x_{n}\right)$ given in $[3$, (2.3)] can be computed as

$$
s_{i, j}=f_{x_{i}} f_{x_{j}} \hat{g}_{00}+f_{x_{i}} \hat{g}_{0 j}+f_{x_{j}} \hat{g}_{i 0}+\hat{g}_{i j} \quad(i, j=1, \ldots, n) .
$$

Since $\left\{\hat{g}_{\alpha \beta}\right\}_{\alpha, \beta=0}^{n}$ are functions of $f$, each $s_{i, j}$ is also a function of $f$ and $f_{I}$, where

$$
f_{I}:=\left\{f_{x_{i}}\right\}_{i=1}^{n} \text {. }
$$

In particular, $B$ is a function of $f$ and $f_{I}$. Also each component $\tilde{s}^{i, j}(i, j,=1, \ldots, n)$ of the cofactor matrix of the $n \times n$-matrix $\left(s_{i, j}\right)$ is also a function of $f$ and $f_{I}$.

By (1.8), $\mu_{i}^{\beta}(i=1, \ldots, n, \beta=0, \ldots, n)$ in [3, Page 3410] are given by

$$
\mu_{i}^{\beta}= \begin{cases}1 & \text { if } \beta=i, \\ f_{x_{i}} & \text { if } \beta=0 \\ 0 & \text { if } \beta \neq 0, i\end{cases}
$$

and

$$
\mu_{i, \beta}=\sum_{\alpha=0}^{n} \mu_{i}^{\alpha} \hat{g}_{\alpha \beta} \quad(i=1, \ldots, n, \beta=0, \ldots, n)
$$

are functions of $f$ and $f_{I}$. In particular, each component $\tilde{\nu}_{i}$ of the normal vector field $\tilde{\nu}$ (cf. [3, (2.5)]) is also a function of $f$ and $f_{I}$. We denote by $\left\{\Gamma_{\alpha \beta}^{\gamma}\right\}_{\alpha, \beta, \gamma=0}^{n}$ the Christoffel symbols of $g$ with respect to the coordinate system $\left(x_{0}, \ldots, x_{n}\right)$ of $M$, and set

$$
\hat{\Gamma}_{\alpha \beta}^{\gamma}:=\Gamma_{\alpha \beta}^{\gamma} \circ F \quad(\alpha, \beta, \gamma=0, \ldots, n),
$$


each of which can be considered as a function of $f$. Since $F_{x_{j}}=f_{x_{j}} \partial_{x_{0}}+\partial_{x_{j}}$, we can write

$$
D_{\partial_{x_{i}}} F_{x_{j}}=f_{x_{i} x_{j}} \partial_{x_{0}}+\sum_{\alpha=0}^{n}\left(f_{x_{i}} f_{x_{j}} \hat{\Gamma}_{00}^{\alpha}+f_{x_{i}} \hat{\Gamma}_{0 j}^{\alpha}+f_{x_{j}} \hat{\Gamma}_{i 0}^{\alpha}+\hat{\Gamma}_{i j}^{\alpha}\right) \partial_{x_{\alpha}},
$$

where $D$ is the Levi-Civita connection induced by $g$. In particular, by [3, (2.6)], $A$ is also a function of $f, f_{I}, f_{I J}$ and $f_{I n}$, where

$$
f_{I J}:=\left\{f_{x_{i} x_{j}}\right\}_{i, j=1}^{n-1}, \quad f_{I n}:=\left\{f_{x_{i} x_{n}}\right\}_{i=1}^{n-1} .
$$

As a consequence, $\tilde{A}$ (cf. (1.7)) has the following expression:

$$
\tilde{A}=\mathcal{R}\left(f, f_{I}\right) f_{x_{n} x_{n}}+\mathcal{S}\left(f_{I}, f_{I J}, f_{I n}, \varphi\right),
$$

where $\mathcal{R}$ is a function of $f, f_{I}$, and $\mathcal{S}$ is a function of $f, f_{I}, f_{I J}, f_{I n}$ and $\varphi$. For example, if $M=\boldsymbol{R}_{1}^{n+1}$ and $\varphi=0$, then $\tilde{A}=A$ and

$$
\begin{aligned}
& \mathcal{R}=1-\sum_{j=1}^{n-1} f_{x_{j}}^{2}, \\
& \mathcal{S}=\sum_{k=1}^{n-1}\left(1-f_{x_{n}}^{2}-\sum_{j=1, j \neq k}^{n-1} f_{x_{j}}^{2}\right) f_{x_{k} x_{k}} \\
& \quad+2 \sum_{1 \leq j<k \leq n-1} f_{x_{j}} f_{x_{k}} f_{x_{j} x_{k}}+2 \sum_{j=1}^{n-1} f_{x_{j}} f_{x_{n}} f_{x_{j} x_{n}} .
\end{aligned}
$$

Although $f_{I}$ (resp. $f_{I J}$ and $f_{I n}$ ) has (resp. have) only $C^{2}$-differentiability (resp. $C^{1}$-differentiability), $\mathcal{R}$ and $\mathcal{S}$ depend smoothly on variables $f, f_{I}, f_{I J}, f_{I n}$ and $\varphi$, because $M$ itself is a $C^{\infty}$-manifold. Differentiating (1.9), we have the following expression of the derivative $\tilde{A}_{x_{i}}$ with respect to $\partial_{x_{i}}$ :

$$
\tilde{A}_{x_{i}}=\mathcal{R}\left(f, f_{I}\right) f_{x_{n} x_{n} x_{i}}+\mathcal{T}\left(f_{I}, f_{I J}, f_{I n}, f_{I J K}, f_{I J n}, \varphi, \varphi_{I}\right),
$$

where $\mathcal{T}$ is a function of $f_{I}, f_{I J}, f_{I n}, f_{I J K}, f_{I J n}, \varphi, \varphi_{I}$ and

$$
f_{I J K}:=\left\{f_{x_{i} x_{j} x_{k}}\right\}_{i, j=1}^{n-1}, \quad f_{I J n}:=\left\{f_{x_{n} x_{i} x_{j}}\right\}_{i, j=1}^{n-1}
$$

For example, if $n=2, \mathcal{R}$ is a function of $f, f_{x_{1}}$ and $\mathcal{S}$ is a function of $\varphi, f, f_{x_{1}}$, $f_{x_{1} x_{1}}, f_{x_{1} x_{2}}$. So we have the following:

$$
\begin{aligned}
& A_{x_{1}}=\mathcal{R}_{f}\left(f, f_{x_{1}}\right) f_{x_{1}} f_{x_{2} x_{2}}+\mathcal{R}_{f_{x_{1}}}\left(f, f_{x_{1}}\right) f_{x_{1} x_{1}} f_{x_{2} x_{2}}+\mathcal{S}_{\varphi}\left(f, f_{x_{1}}, f_{x_{1} x_{1}}, f_{x_{1} x_{2}}, \varphi\right) \varphi_{x_{1}} \\
& \quad+\mathcal{S}_{f}\left(f, f_{x_{1}}, f_{x_{1} x_{1}}, f_{x_{1} x_{2}}, \varphi\right) f_{x_{1}}+\mathcal{S}_{f_{x_{1}}}\left(f, f_{x_{1}}, f_{x_{1} x_{1}}, f_{x_{1} x_{2}}, \varphi\right) f_{x_{1} x_{1}} \\
& \quad+\mathcal{S}_{f_{x_{1} x_{1}}}\left(f, f_{x_{1}}, f_{x_{1} x_{1}}, f_{x_{1} x_{2}}, \varphi\right) f_{x_{1} x_{1} x_{1}}+\mathcal{S}_{f_{x_{1} x_{2}}}\left(f, f_{x_{1}}, f_{x_{1} x_{1}}, f_{x_{1} x_{2}}, \varphi\right) f_{x_{1} x_{2} x_{1}}
\end{aligned}
$$

where, for example,

$$
\mathcal{R}_{f}\left(f, f_{x_{1}}\right):=\frac{\partial \mathcal{R}\left(f, f_{x_{1}}\right)}{\partial f}, \quad \mathcal{R}_{f_{x_{1}}}\left(f, f_{x_{1}}\right):=\frac{\partial \mathcal{R}\left(f, f_{x_{1}}\right)}{\partial f_{x_{1}}} .
$$

Since $\tilde{A}$ vanishes identically, we have $\tilde{A}=0$ and $\tilde{A}_{x_{i}}=0$ for $i=1, \ldots, n-1$. We set $\boldsymbol{x}:=\left(x_{1}, \ldots, x_{n-1}\right)$ and substitute $\boldsymbol{x}=(0, \ldots, 0)$ to them. Then they are functions of one variable $y$, and we have

$$
\begin{aligned}
& \left.(\mathfrak{a}:=) \tilde{A}\right|_{\boldsymbol{x}=(0, \ldots, 0)}=0, \\
& \left.\left(\mathfrak{a}_{i}:=\right) \tilde{A}_{x_{i}}\right|_{\boldsymbol{x}=(0, \ldots, 0)}=0 \quad(i=1, \ldots, n-1) .
\end{aligned}
$$


Although $\mathfrak{a}$ and $\mathfrak{a}_{i}(i=1, \ldots, n-1)$ are all functions of $y$, we now would like to think that they are functions of $a, a^{\prime}, a^{\prime \prime}, b_{I}, b_{I}^{\prime}, b_{I}^{\prime \prime}, c_{I J}, c_{I J K}$ and $\varphi$, where

$$
\begin{aligned}
& c_{i j}(y):=c_{x_{i} x_{j}}(0, \ldots, 0, y), \quad c_{i j k}(y):=c_{x_{i} x_{j} x_{k}}(0, \ldots, 0, y), \\
& \hat{\varphi}(y):=\varphi(0, \ldots, 0, y), \quad \hat{\varphi}_{i}(y):=\varphi_{x_{i}}(0, \ldots, 0, y)
\end{aligned}
$$

and

$$
\begin{aligned}
& b_{I}:=\left\{b_{i}\right\}_{i=1}^{n-1}, \quad b_{I}^{\prime}:=\left\{b_{i}^{\prime}\right\}_{i=1}^{n-1}, \quad b_{I}^{\prime \prime}:=\left\{b_{i}^{\prime \prime}\right\}_{i=1}^{n-1}, \\
& \hat{\varphi}_{I}:=\left\{\hat{\varphi}_{i}\right\}_{i=1}^{n-1}, \quad c_{I J}:=\left\{c_{i j}\right\}_{i, j=1}^{n-1}, \quad c_{I J K}=\left\{c_{i j k}\right\}_{i, j, k=1}^{n-1} .
\end{aligned}
$$

Then we have the following expressions

$$
\begin{aligned}
\mathfrak{a}= & \Lambda^{1}\left(a, a^{\prime}, b_{I}, b_{I}^{\prime}, c_{I J}, c_{I J K}, \hat{\varphi}\right) a^{\prime \prime}+\Lambda^{2}\left(a, a^{\prime}, b_{I}, b_{I}^{\prime}, c_{I J}, c_{I J K}, \hat{\varphi}\right), \\
\mathfrak{a}_{i}= & \Lambda_{i}^{3}\left(a, a^{\prime}, b_{I}, b_{I}^{\prime}, c_{I J}, c_{I J K}, \hat{\varphi}, \hat{\varphi}_{I}\right) a^{\prime \prime} \\
& +\Lambda_{i}^{4}\left(a, a^{\prime}, b_{I}, b_{I}^{\prime}, c_{I J}, c_{I J K}, \hat{\varphi}, \hat{\varphi}_{I}\right) b_{i}^{\prime \prime}+\Lambda_{i}^{5}\left(a, a^{\prime}, b_{I}, b_{I}^{\prime}, c_{I J}, c_{I J K}, \hat{\varphi}, \hat{\varphi}_{I}\right),
\end{aligned}
$$

where $\Lambda^{1}, \Lambda^{2}$ and $\Lambda_{i}^{l}(l=3, \ldots, 5, i=1, \ldots, n-1)$ are functions which are $C^{\infty}$ differentiable with respect to the parameters $a, a^{\prime}, b_{I}, b_{I}^{\prime}, c_{I J}, c_{I J K}, \hat{\varphi}, \hat{\varphi}_{I}$. For example, the explicit expressions of $\mathfrak{a}$ and $\mathfrak{a}_{i}$ for $M=\boldsymbol{R}_{1}^{3}$ are given in 4, (4.4) and $(4.5)]$.

Since $\mathcal{R}=1$ at $o, \Lambda^{1}$ and $\Lambda_{i}^{4}(i=1, \ldots, n-1)$ do not vanish at $o$ under the initial condition (1.6). Regarding $c_{I J}, c_{I J K}, \hat{\varphi}$ and $\hat{\varphi}_{I}$ are functions of $y$, we can rewrite (1.12) and (1.13) in the following forms

$$
\begin{aligned}
& a^{\prime \prime}=P\left(y, a, a^{\prime}, b_{I}, b_{I}^{\prime}\right), \\
& b_{i}^{\prime \prime}=Q_{i}\left(y, a, a^{\prime}, b_{I}, b_{I}^{\prime}\right) \quad(i=1,2, \ldots, n-1),
\end{aligned}
$$

where $P, Q_{i}$ are functions of $y, a, a^{\prime}, b_{I}, b_{I}^{\prime}$.

For example, we consider the case $M=\boldsymbol{R}_{1}^{n+1}$. If we set

$$
\begin{gathered}
c_{i j}(y):=c_{x_{i} x_{j}}(0, \ldots, 0, y), \quad c_{i j k}(y):=c_{x_{i} x_{j} x_{k}}(0, \ldots, 0, y), \\
c_{i j}^{\prime}(y):=c_{x_{i} x_{j} y}(0, \ldots, 0, y),
\end{gathered}
$$

then

$$
\begin{aligned}
\alpha & =C a^{\prime \prime}+\sum_{j=1}^{n-1} c_{j j}\left(D+b_{j}^{2}\right)+2 \sum_{i<j} b_{i} b_{j} c_{i j}+2 \sum_{i=1}^{n-1} a^{\prime} b_{i} b_{i}^{\prime} \\
\alpha_{l} & =C b_{l}^{\prime \prime}-2\left(\sum_{j=1}^{n-1} b_{j} c_{j l}\right) a^{\prime \prime}+\sum_{j=1}^{n-1}\left(D+b_{j}^{2}\right) c_{j j l} \\
& -2 \sum_{j=1}^{n-1}\left(a^{\prime} b_{l}^{\prime}+\sum_{i=1, i \neq j}^{n-1} b_{i} c_{i l}\right) c_{j j}+2 \sum_{i<j}\left(b_{j} c_{i l} c_{i j}+b_{i} c_{j l} c_{i j}+b_{i} b_{j} c_{i j l}\right) \\
& +2 \sum_{i=1}^{n-1}\left(c_{i l} a^{\prime} b_{i}^{\prime}+b_{i} b_{l}^{\prime} b_{i}^{\prime}+b_{i} a^{\prime} c_{i l}^{\prime}\right)
\end{aligned}
$$

hold for $l=1, \ldots, n-1$, where $C:=1-\sum_{i=1}^{n-1} b_{i}^{2}$ and $D:=1-\left(a^{\prime}\right)^{2}-\sum_{i=1}^{n-1} b_{i}^{2}$.

In particular, (1.16) and (1.17) give a normal form for a system of ordinary differential equations thinking $a, b_{i}(i=1, \ldots, n-1)$ are unknown functions, and can be considered as precise expressions of [3, (4.12)]. Since $P, Q_{i}(i=1, \ldots, n-1)$ are $C^{\infty}$-differentiable with respect to the variables $a, a^{\prime}, b_{I}, b_{I}^{\prime}$, this system of ordinary differential equations satisfies the local Lipschitz condition. So the uniqueness of the solution follows. On the other hand, by applying [3, Proposition 4.2],

$$
a(y)=y, \quad b_{i}(y)=0 \quad(i=1, \ldots, n-1)
$$


gives a solution of this system satisfying the initial condition (1.6). However, we should remark that in the proof of [3, Proposition 4.2], we set (cf. (4.14))

$$
f_{0}\left(x_{1}, \ldots, x_{n}\right):=x_{n}+\sum_{1 \leq j \leq k \leq n-1} c_{j, k}\left(x_{1}, \ldots, x_{n}\right) x_{j} x_{k} .
$$

However, by the same reason as Remark 1, we should replace it by

$$
f_{0}\left(x_{1}, \ldots, x_{n}\right):=x_{n}+c\left(x_{1}, \ldots, x_{n}\right),
$$

where $c$ is a $C^{3}$-function satisfying (1.3) and (1.4). Then

$$
x_{n} \mapsto\left(f_{0}\left(0, \ldots, 0, x_{n}\right), 0, \ldots, 0, x_{n}\right)
$$

gives a light-like geodesic of $M$ consisting of degenerate light-like points.

So, we can conclude that (1.6) implies (1.20), that is, we have proven the theorem.

Remark. We point out that there is a minor typographical error in the proof of Theorem D in Section 6 in 3 . In fact, in the beginning of the proof, we had set $f(x, y)=a(y)+b(y) x+h(x, y) x^{3}$, but it should be $f(x, y)=a(y)+b(y) x+h(x, y) x^{2}$. The remaining arguments can be read without any need for corrections.

Acknowledgements. The authors thank Professor Udo Hertrich-Jeromin for valuable comments.

\section{REFERENCES}

[1] S. Akamine, A. Honda, M. Umehara and K. Yamada, Bernstein-type theorem for zero mean curvature hypersurfaces without time-like points in Lorentz-Minkowski space, Bull. Braz. Math. Soc. (N.S.), (2020), DOI 10.1007/s00574-020-00196-8.

[2] V. A. Klyachin, Zero mean curvature surfaces of mixed type in Minkowski space, Izv. Math., 67 (2003), 209-224.

[3] M. Umehara and K. Yamada, Hypersurfaces with light-like points in a Lorentzian manifold, J. Geom. Anal., 29 (2019), 3405-3437.

[4] M. Umehara and K. Yamada, Surfaces with light-like points in Lorentz-Minkowski space with applications, in "Lorentzian Geometry and Related Topics", Springer Proceedings of Mathematics \& Statics 211, 2017, pp. 253-273, Springer-Verlag.

(Umehara) Department of Mathematical and Computing Sciences, Tokyo Institute of TECHNOLOGY, TOKYO 152-8552, JAPAN

E-mail address: umehara@is.titech.ac.jp

(Yamada) Department of Mathematics, Tokyo Institute of Technology, Tokyo 1528551 , JAPAN

E-mail address: kotaro@math.titech.ac.jp 